\title{
Methylene Blue Loaded PLGA Nanoparticles: Combined Emulsion, Drug Release Analysis and Photodynamic Activity
}

\author{
Cindy Alejandra Gutiérrez-Valenzuela ${ }^{1}$, Rosalva Rodríguez-Córdova ${ }^{1}$, Yesenia Hernández-Giottonini ${ }^{1}$, \\ Patricia Guerrero-Germán ${ }^{2}$, Armando Lucero-Acuña ${ }^{2}$, \\ 1. Nanotechnology Graduate Program, Department of Physics, University of Sonora, Hermosillo, Mexico \\ 2. Department of Chemical and Metallurgical Engineering, University of Sonora, Hermosillo, Mexico
}

The use of polymeric nanoparticles for the control release of photosensitizer compounds such as methylene blue represents a promising option for cancer treatment. Methylene blue (MB) has been of great interest in many areas of clinical medicine, from neurological disorders to cancer chemotherapy [12]. It can be used in photodynamic therapy, which consists on the application of $\mathrm{MB}$ in the area of interest, and then activated by light at $665 \mathrm{~nm}$ producing reactive oxygen species that leads to the death of the target cell via oxidative damage. Polymeric nanoparticles loaded with methylene blue (MB-PNP) were prepared by using a combined emulsification technique [4-5]. Briefly, $\mathrm{MB}$ is dissolved in deionized water and into a DCM solution containing MB and PLGA. The mixture is emulsified at $22 \%$ of amplitude $(26.5 \mu \mathrm{m})$ by sonication. Next, an aqueous solution of $5 \% \mathrm{w} / \mathrm{v}$ PVA is added into the mixture and a second emulsification is carried during at $75 \%$ amplitude $(90 \mu \mathrm{m})$. The solvent is evaporated under magnetic stirring, at room temperature. MB-PNPs are washed by three centrifugation cycles and freeze-dried for further characterization. All experiments were performed by triplicate.

Nanoparticle size distribution and zeta potentials were measured using a zetasizer Nano ZS equipment (Malvern) by dynamic light scattering and laser Doppler electrophoresis, respectively. Average values of particle size obtained were $180 \mathrm{~nm}$ with 0.030 polydispersity index (PDI) and $190 \mathrm{~nm}$ with 0.109 PDI for blank PNP and 12\% TDL MB-PNP respectively. Zeta potential values obtained were $-31.2 \mathrm{mV}$ for blank-PNP and $-18.2 \mathrm{mV}$ for MB-PNP. This difference in superficial charge can be attributed to the presence of $\mathrm{MB}$ on the surface of the nanoparticle. Similar work has been reported in literature where MB is encapsulated in PLGA using different encapsulation methods, and sizes range from 190 to 220 $\mathrm{nm}$, with zeta potentials ranging between $-38 \mathrm{mV}$ and $-17.5 \mathrm{mV}$ [3]. When encapsulating $2.5 \%$ TDL, authors have obtained 220 to $266 \mathrm{~nm}$ diameters with PDI values of 0.19 and 0.4 [1]. Other materials have also been used to encapsulate MB such as silica nanoparticles, resulting in diameters of particle of $105 \mathrm{~nm}$, with zeta potentials between -44 and $-29 \mathrm{mV}$ [5]. Surface morphology of MB-PNP was analysed by scanning electron microscopy (JEOL) (Figure 1). The drug loading (DL) for MB-PNP was $0.97 \%$, resulting in a $8.06 \%$ encapsulation efficiency (EE). Cannavà et al. obtained drug loadings values of $0.52 \%$ to $1.13 \%$ and encapsulation efficiencies of $3.13 \%$ to $6.75 \%$ [1]. These values are comparable to the ones obtained in this work. Encapsulation efficiency depends on the amount of drug used at the beginning of the formulation, meaning that less MB was used in the formulation in order to obtain the drug load desired.

To evaluate in vitro MB release from MB-PNPs, the dialysis method is used [1]. MB concentrations were quantified by spectrometry at $665 \mathrm{~nm}$ using a calibration curve. During the study, the amount of MB released in the initial burst stage was $21 \mu \mathrm{g}$, which correspond to the $80 \%$ within the first 4 hours. This can be attributed to the high solubility nature of the MB. The assumption is that most of the drug was encapsulated close to the surface of nanoparticles; this could explain the high rate of release in the initial burst by rapid dissolution of $\mathrm{MB}$ in the release media. After this stage, a slower release was 
observed for 48 hours until a plateau was reached indicating that all MB was released from the nanoparticles. This slower release can be explained by the diffusion of the MB through the polymer matrix into the release media. This same conduct is reported in literature, where $80 \%$ of encapsulated MB was release after 5-12 hours, reaching a $100 \%$ at approximately 24 hours $[1,6]$.

The photodynamic activity was measured by irradiating MB solution with red light (660 nm; 100 $\mathrm{mW} / \mathrm{cm}^{2}$ ) for 20 seconds in intervals of 2 seconds. Diphenylisobenzofuran (DPBF) was used as a chemical probe for ${ }^{1} \mathrm{O}_{2}[8]$.

\section{References:}

[1] C. Cannavà et al, RSC Adv 6 (2016), p. 16720.

[2] M. Wainwright and K.B. Crossley, Journal of Chemotherapy 14 (2002), p. 431

[3] H.C. Huang, Nanomedicine 6 (2011), p. 459.

[4] S.H.S Boddu and R. Vaishya, Medicinal Chemistry 2 (2012), p. 068.

[5] Danhier, E. Ansorena, J.M. Silva, Journal of Controlled Release 161 (2012), p. 505

[6] V. Klepac-Ceraj et al, Lasers in Surgery and Medicine 43 (2011), p. 600.

[7] X. He et al, Biomaterials 30 (2009), p. 5601.

[8] The authors acknowledge funding from CONACYT with the projects I0007-2013-02 No. 206489 and INFR-2014-01 No. 226208. Also financial assistance is acknowledged to the Secretariat of Public Education of Mexico (SEP) with the project UNISON-PTC-222 DSA/103.5/15/7356

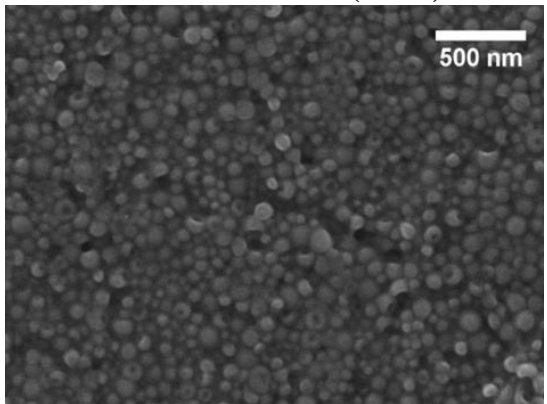

Figure 1. SEM images of blank-PNP of approximately $180 \mathrm{~nm}$.

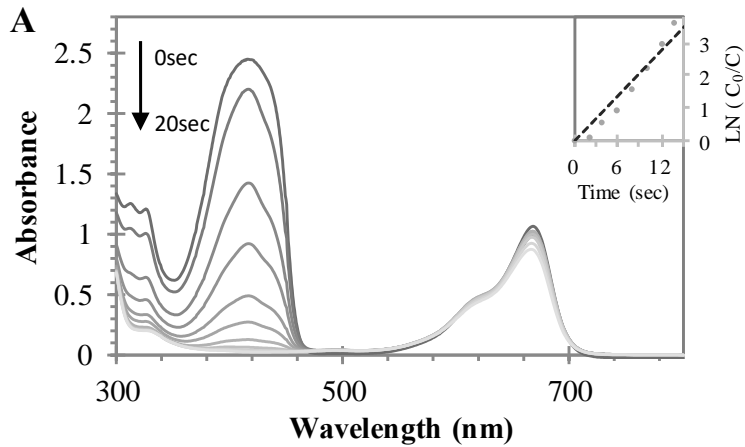

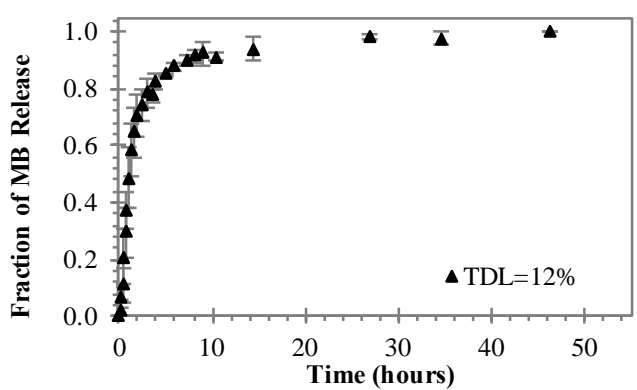

Figure 2. In vitro $\mathrm{MB}$ release profiles $12 \% \mathrm{TDL}$ (A) prepared by a combined W/O/W emulsion method. Data represent mean $\pm \mathrm{SD}(\mathrm{n}=3)$.

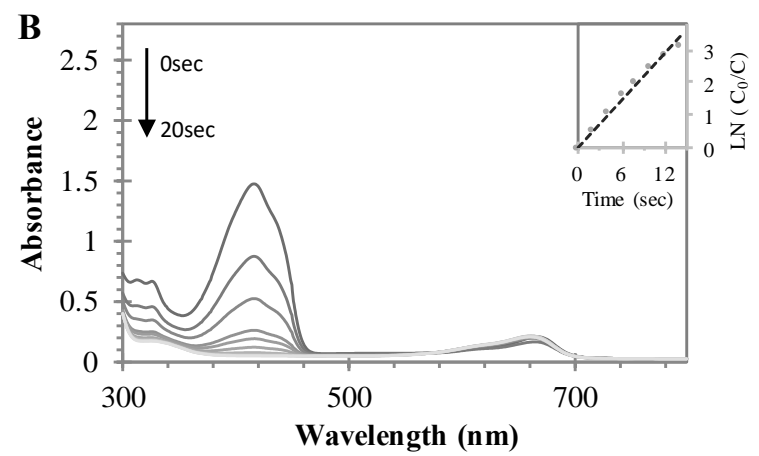

Figure 3. DPBF photodegradation as result of single oxygen production when (A) $5 \mathrm{mg} / \mathrm{mL} \mathrm{MB}$ solution and (B) MB-PNP after four hours of release are irradiated with a red laser at $660 \mathrm{~nm}$ wavelength with $100 \mathrm{~mW} / \mathrm{cm}^{2}$. 